\title{
Interactive comment on "Anoxic iron and sulphur cycling in the cGENIE.muffin Earth system model (v0.9.16)" by Sebastiaan J. van de Velde et al.
}

\section{Anonymous Referee \#2}

Received and published: 8 December 2020

The modeling of pelagic Fe-S dynamics presented in the paper is a useful addition to the existing GENIE model framework. The reaction network developed by the authors considers most of the key processes involved in pelagic Fe-S cycling under anoxic conditions. I only found one model equation (Eq. 16, R7) that is probably not correct. In this equation, it is assumed that ferric iron oxidizes dissolved sulfide to sulfate while the evaluable data show that elemental sulfur is the major product of anaerobic sulfide oxidation with ferric iron oxides (e.g. Poulton et al. 2004). The elemental sulfur formed during this reaction is subsequently converted into pyrite by further reactions with sulfide and iron minerals under anoxic conditions. Since it would be difficult to introduce elemental sulfur as an additional tracer, I would recommend to use a more realistic stoichiometry for $\mathrm{R} 7$ where sulfide is converted into pyrite rather than sulfate. The au-

Printer-friendly version

Discussion paper 
thors should at least show one additional model run with this revised stoichiometry to investigate to what extent the model output is affected by the artificial sulfate source introduced via R7.

The other problem of the paper is related to the lack of validation. The authors acknowledge that key processes such as the benthic iron cycle and the pelagic/benthic nitrogen cycle are not considered in their modeling framework. Since these processes play important roles, both in ancient oceans and modern lakes, it seems to be impossible to validate the model by field observations and proxy records. I do understand that the authors plan to expand their model to include these processes and that they see their manuscript as a step that needs to be taken to produce a more complete GENIE model version. To demonstrate the potential importance of their contribution, the authors should consider to embed their new pelagic $\mathrm{Fe} / \mathrm{S}$ machinery into published box models that consider benthic Fe/S and nitrogen cycling to test how the performance of these models is enhanced by their pelagic Fe/S model equations.

The paper is well written and potentially useful. It could be further improved during the revision process by considering the comments above.

Interactive comment on Geosci. Model Dev. Discuss., https://doi.org/10.5194/gmd-2020-312, 2020. 